\title{
On vertex operator realizations of Jack functions
}

\author{
Wuxing Cai · Naihuan Jing
}

Received: 21 December 2009 / Accepted: 29 March 2010 / Published online: 20 April 2010

(C) Springer Science+Business Media, LLC 2010

\begin{abstract}
On the vertex operator algebra associated with a rank one lattice we derive a general formula for products of vertex operators in terms of generalized homogeneous symmetric functions. As an application we realize Jack symmetric functions of rectangular shapes as well as marked rectangular shapes.
\end{abstract}

Keywords Symmetric functions · Jack polynomials · Vertex operators

\section{Introduction}

Classical symmetric functions play important roles in many areas of mathematics and physics, and in particular they have close ties with representation theory of both finite and infinite dimensional Lie algebras. Starting with Bernstein's work (see [15]), vertex operators have been used in constructing several families of symmetric functions such as Schur and Schur's Q-functions [4] as well as Hall-Littlewood symmetric functions [5]. Though one can still define a certain family of vertex operators associated with Macdonald polynomials similar to the Schur case, the products of the vertex operators are in general no longer equal to the Macdonald polynomials. At least in the case of two rows the transition function from the basis of generalized homogeneous

N. Jing gratefully acknowledges the support from NSF as well as NSFC Grant (10801094).

W. Cai $\cdot$ N. Jing

School of Sciences, South China University of Technology, Guangzhou 510640, China

W. Cai

e-mail: caiwx@scut.edu.cn

N. Jing (凶)

Department of Mathematics, North Carolina State University, Raleigh, NC 27695, USA

e-mail: jing@ math.ncsu.edu 
symmetric functions (product of one-row Mcdonald polynomials) involves with hypergeometric series of type ${ }_{4} \phi_{3}$. Except for a few cases $[6,14]$, it has been an open problem to find the transition function from the generalized homogeneous functions to Macdonald symmetric functions [2, 6].

On the other hand, in the study of vertex representations of affine Lie algebras, Lepowsky and Wilson [9] have long posted the important problem on whether certain products in the representation space are linearly independent. In the special case of level three representations, this problem can be solved using Rogers-Ramanujan identities (see also [8] for the homogeneous case). Later it was realized [6] that the vertex operators at level three are actually related with vertex operators associated to certain Jack polynomials, namely, the half vertex operators are actually the generating function of the one-row Jack functions. Thus it is also an interesting question to study the linear independence problem for those vertex operators associated to Jack functions.

Motivated by [1] we define the vertex operator associated with Jack functions in the vertex operator algebra of rank one lattice. They are called Jack vertex operator since the product of identical modes of the vertex operator will be shown to be Jack functions of rectangular shapes. It is interesting that the contraction functions for products of the new vertex operator are of the form $\prod_{i<j}\left(z_{i}-z_{j}\right)^{2 \alpha}$ instead of $\prod_{i<j}\left(z_{i}-z_{j}\right)^{\alpha}$ for the Jack parameter $\alpha^{-1}$ (which are for $Y_{1}(z)$ in Sect. 3.2), as expected from experience with the Schur and Hall-Littlewood cases. It turns out that one really needs this new form of vertex operators (vertex operator $X(z)$ in Sect. 3.2) to generate rectangular Jack functions. At the special case of Schur functions $(\alpha=1)$, our new vertex operators provide another formula for the rectangular shapes.

We also study the problem of linear independence for the new vertex operators in the case of Jack functions. We show that under certain conditions the set of vertex operator products is indeed a basis for the representation space (see [1] for a similar statement). We achieve this by deriving a Jacobi-Trudi like formula for the Jack vertex operator, and then we reprove Mimachi-Yamada's theorem [11] that the product of the vertex operators are Jack functions for the rectangular shapes, and then we further generalize this formula to the case of marked rectangular shapes, i.e., rectangular shapes minus part of the last column. This general case contains part of [7] as a special case.

This paper is organized as follows. In Sect. 2 we recall some necessary notions of symmetric functions. In Sect. 3 we first review the vertex operator approach based on the second author's work on Schur functions, then we define the Jack vertex operators and give an explicit formula of the vertex operator products and a Jacobi-Trudi like formula in terms of tableaux. In Sect. 4 we provide a detailed analysis of certain matrix coefficients of vertex operators and prove the theorem of realizing Jack functions of rectangular and marked rectangular shapes.

\section{Jack functions}

We recall some basic notions about symmetric functions following the standard reference [10]. A partition $\lambda$ is a sequence $\lambda=\left(\lambda_{1}, \lambda_{2}, \ldots, \lambda_{s}\right)$ of nonnegative integer such that $\lambda_{1} \geq \lambda_{2} \geq \cdots \geq \lambda_{s}$; the set of all partitions is denoted by $\mathcal{P}$; we 
sometimes write $\lambda$ as $\left(1^{m_{1}} 2^{m_{2}} \cdots\right)$, where $m_{i}$ is the multiplicity of $i$ occurring in the parts of $\lambda$. The number of non-zero $\lambda_{i}$ 's is called the length of $\lambda$, denoted by $l(\lambda)$, and the weight $|\lambda|$ is defined as $\lambda_{1}+\cdots+\lambda_{s}$. A partition $\lambda$ of weight $n$ is usually denoted by $\lambda \vdash n$. We also recall that the dominance order is defined by comparing the partial sums of the parts. For two partitions $\lambda$ and $\mu$ of the same weight, if $\lambda_{1}+\cdots+\lambda_{i} \geq \mu_{1}+\cdots+\mu_{i}$ for all $i$, one says that $\lambda$ is greater than $\mu$ and denoted as $\lambda \geq \mu$; conventionally, $\lambda>\mu$ means $\lambda \geq \mu$ but $\lambda \neq \mu$. For $\lambda=\left(\lambda_{1}, \lambda_{2}, \ldots\right)=\left(1^{m_{1}}, 2^{m_{2}}, \ldots\right), \mu=\left(\mu_{1}, \mu_{2}, \ldots\right)=\left(1^{n_{1}}, 2^{n_{2}}, \ldots\right)$ the notation $\lambda-\mu$ means $\left(\lambda_{1}-\mu_{1}, \lambda_{2}-\mu_{2}, \ldots\right), \mu \subset^{\prime} \lambda$ means that $n_{1} \leq m_{1}, n_{2} \leq m_{2}, \ldots$, and $\lambda \backslash \mu$ denotes the partition $\left(1^{m_{1}-n_{1}} 2^{m_{2}-n_{2}} \cdots\right)$. We also define $\left(\begin{array}{c}m(\lambda) \\ m(\mu)\end{array}\right)=\left(\begin{array}{c}m_{1} \\ n_{1}\end{array}\right)\left(\begin{array}{c}m_{2} \\ n_{2}\end{array}\right) \cdots$, and $\lambda \cup \mu=\left(\begin{array}{lll}1^{m_{1}+n_{1}} & \left.2^{m_{2}+n_{2}} \cdots\right)\end{array}\right)$.

The ring $\Lambda$ of symmetric functions over $\mathbb{Z}$ has various linear $\mathbb{Z}$-bases indexed by partitions: the monomial symmetric functions $m_{\lambda}=\sum x_{i_{1}}^{\lambda_{1}} \cdots x_{i_{k}}^{\lambda_{k}}$, the elementary symmetric functions $e_{\lambda}=e_{\lambda_{1}} \cdots e_{\lambda_{k}}$ with $e_{n}=m_{\left(1^{n}\right)}$, and the Schur symmetric functions $s_{\lambda}$. The power sum symmetric functions $p_{\lambda}=p_{\lambda_{1}} \cdots p_{\lambda_{k}}$ form a $\mathbb{Q}$-basis.

Let $F=\mathbb{Q}(\alpha)$ be the field of rational functions in indeterminate $\alpha$. The Jack polynomial [3] is a special orthogonal symmetric function under the following inner product. For two partitions $\lambda, \mu \in \mathcal{P}$ the scalar product on $\Lambda_{F}$ is given by

$$
\left\langle p_{\lambda}, p_{\mu}\right\rangle=\delta_{\lambda, \mu} \alpha^{-l(\lambda)} z_{\lambda}
$$

where $z_{\lambda}=\prod_{i \geq 1} i^{m_{i}} m_{i} !, m_{i}$ is the occurrence of integer $i$ in the partition $\lambda$, and $\delta$ is the Kronecker symbol. Here our parameter $\alpha$ is chosen as the reciprocal to the usual convention in view of our vertex operator realization.

In [10] Macdonald proved the existence and uniqueness of what is called the Jack function as a distinguished family of orthogonal symmetric functions $P_{\lambda}\left(\alpha^{-1}\right)$ with respect to the scalar product (2.1) in the following sense:

$$
P_{\lambda}\left(\alpha^{-1}\right)=\sum_{\lambda \geq \mu} c_{\lambda \mu}\left(\alpha^{-1}\right) m_{\mu}
$$

in which $c_{\lambda \mu}\left(\alpha^{-1}\right) \in F, \lambda, \mu \in \mathcal{P}$, and $c_{\lambda \lambda}\left(\alpha^{-1}\right)=1$. Let

$$
b_{\lambda}\left(\alpha^{-1}\right)=\left\langle P_{\lambda}, P_{\lambda}\right\rangle^{-1},
$$

then $Q_{\lambda}\left(\alpha^{-1}\right)=b_{\lambda}\left(\alpha^{-1}\right) P_{\lambda}\left(\alpha^{-1}\right)$ is the dual Jack function.

It is known that the special case $Q_{(n)}\left(\alpha^{-1}\right)$, simplified as $Q_{n}\left(\alpha^{-1}\right)$, can be written explicitly:

$$
Q_{n}\left(\alpha^{-1}\right)=\sum_{\lambda \vdash n} \alpha^{l(\lambda)} z_{\lambda}^{-1} p_{\lambda} .
$$

For a partition $\lambda$, we will denote $q_{\lambda}\left(\alpha^{-1}\right)=Q_{\lambda_{1}}\left(\alpha^{-1}\right) Q_{\lambda_{2}}\left(\alpha^{-1}\right) \cdots Q_{\lambda_{l}}\left(\alpha^{-1}\right)$.

According to Stanley [13] the $q_{\lambda}$ 's also form another basis of $\Lambda_{F}$, and they are dual to that of $m_{\lambda}$. Hence the transition matrix from $Q_{\lambda}$ 's to $q_{\lambda}$ 's is the transpose of that from $m_{\lambda}$ 's to $P_{\lambda}$ 's. Explicitly, we have 
Lemma 2.1 For any partition $\lambda$, one has

$$
\begin{aligned}
& q_{\lambda}\left(\alpha^{-1}\right)=\sum_{\mu \geq \lambda} c_{\lambda \mu}^{\prime} Q_{\mu}\left(\alpha^{-1}\right), \\
& Q_{\lambda}\left(\alpha^{-1}\right)=\sum_{\mu \geq \lambda} d_{\lambda \mu}^{\prime} q_{\mu}\left(\alpha^{-1}\right),
\end{aligned}
$$

where $d_{\lambda, \mu}^{\prime} \in F$, with $c_{\lambda \mu}^{\prime}=c_{\mu \lambda}$ and $c_{\lambda \lambda}^{\prime}=d_{\lambda \lambda}^{\prime}=1$.

\section{Vertex operators and symmetric functions}

Vertex operators can be used to realize several classical types of symmetric functions such as Schur and Hall-Littlewood polynomials [4, 5]. There are some partial progress towards realizations of Macdonald polynomials [4, 14]. In order to discuss the Jack case, we will use the standard vertex algebra technique and recall the construction of lattice vertex operator algebra for rank one case.

\subsection{Representation space $V$ and transformation to $\Lambda_{C}$}

For a positive integer $\alpha$, the complex Heisenberg algebra $H_{\alpha}=\bigoplus_{n \neq 0} \mathbb{C} h_{n}+\mathbb{C} c$ is the infinite dimensional Lie algebra generated by $h_{n}$ and $c$ subject to the following defining relations:

$$
\left[h_{m}, h_{n}\right]=\delta_{m,-n} \alpha^{-1} m c, \quad\left[h_{m}, c\right]=0 .
$$

We remark that the integer $\alpha$ is included for identification with Jack inner product. If it is clear from the context, we will omit the subscript $\alpha$ in $H_{\alpha}$ and simply refer it as $H$.

It is well known that $H$ has a unique canonical representation given as follows. The representation space can be realized as the infinite dimensional vector space $V_{0}=\operatorname{Sym}\left(h_{-1}, h_{-2}, \ldots\right)$, the symmetric algebra over $\mathbb{C}$, generated by $h_{-1}, h_{-2}, \ldots$ The action of $H$ is given by

$$
\begin{aligned}
h_{n} . v & =\alpha^{-1} n \frac{\partial}{\partial h_{-n}} v \quad \text { if } n>0, \\
h_{n} \cdot v & =h_{n} v \quad \text { if } n<0, \\
c . v & =v .
\end{aligned}
$$

To simplify the indices we enlarge the space $V_{0}$ by the group algebra of $\frac{1}{2} \mathbb{Z}$. Let $V=V_{0} \otimes \mathbb{C}\left[\frac{1}{2} \mathbb{Z}\right]$, where $\mathbb{C}\left[\frac{1}{2} \mathbb{Z}\right]$ is the group algebra of $\frac{1}{2} \mathbb{Z}$ with generators $\left\{e^{n h} \mid\right.$ $\left.n \in \frac{1}{2} \mathbb{Z}\right\}$. We define the action of the group algebra as usual with the multiplication given by $e^{m h} e^{n h}=e^{(m+n) h}$. We also define the action of $\partial=\partial_{h}$ on $\mathbb{C}[Z]$ by д. $e^{m h}=m e^{m h}$. The space $V_{0}$ is $\mathbb{Z}$-graded. The enlarged space $V$ is $\mathbb{Z} \times \frac{1}{2} \mathbb{Z}$-graded as 
follows. Let $\lambda=\left(\lambda_{1}, \lambda_{2}, \ldots\right)$ be a partition, for $v=h_{-\lambda} \otimes e^{n h} \in V$, define the degree of $v$ by $d(v)=(|\lambda|, n)$, where we have used the usual notation $h_{-\lambda}=h_{-\lambda_{1}} h_{-\lambda_{2}} \cdots$. For convenience, we consider the degree of zero element to be of any value.

The vertex operator space $V$ has a canonical scalar product. For any polynomials $P, Q$ in the $h_{i}$ 's we have

$$
\begin{aligned}
\left\langle h_{n} P, Q\right\rangle & =\left\langle P, h_{-n} Q\right\rangle, \\
\langle 1,1\rangle & =1, \\
\left\langle e^{m h}, e^{n h}\right\rangle & =\delta_{m, n} .
\end{aligned}
$$

Thus, for partitions $\lambda, \mu$ we have

$$
\left\langle h_{-\lambda} \otimes e^{m h}, h_{-\mu} \otimes e^{n h}\right\rangle=z_{\lambda} \alpha^{-l(\lambda)} \delta_{\lambda, \mu} \delta_{m, n}
$$

We define a linear map $T: V=\sum_{s \in \frac{1}{2} \mathbb{Z}} V_{s} \mapsto \Lambda_{\mathbb{Q}}$ by:

$$
T: h_{-\lambda} \otimes e^{s h} \mapsto p_{\lambda}
$$

We remark that the restriction of $T$ on $V_{s}=V_{0} \otimes e^{s h}$ is a bijection preserving the products.

\subsection{Jack vertex operator on $V$ and an explicit formula}

For a complex parameter $a$ we let the vertex operator $Y_{a}(z)$ acts on $V$ via the generating series:

$$
Y_{a}(z)=\exp \left(\sum_{n=1}^{\infty} \frac{z^{n}}{n} \alpha h_{-n}\right) \exp \left(\sum_{n=1}^{\infty} \frac{z^{-n}}{-n} a \alpha h_{n}\right)=\sum_{n} Y_{a}(n) z^{-n}
$$

For $\alpha \in \frac{1}{2} \mathbb{Z}$, we define $X(z)=Y_{2}(z) \exp \left(2 \alpha \ln z \partial_{h}+h\right)$, i.e.

$$
X(z)=\exp \left(\sum_{n=1}^{\infty} \frac{z^{n}}{n} \alpha h_{-n}\right) \exp \left(2 \alpha \ln z \partial_{h}+h\right) \exp \left(\sum_{n=1}^{\infty} \frac{z^{-n}}{-n} 2 \alpha h_{n}\right)
$$

where the middle term acts as follows:

$$
\exp \left(2 \alpha \ln z \partial_{h}+h\right) \cdot e^{s h}=z^{\left(s+\frac{1}{2}\right) 2 \alpha} e^{(s+1) h} .
$$

The operator $X_{n}$ on $V$ is defined as the component of $X(z)$ :

$$
X(z)=\sum_{n \in \mathbb{Z}} X_{n} z^{-n}
$$


Clearly for a vector $v$ of degree $(k, m)$, one has

$$
d\left(X_{n} v\right)=(k-n-(2 m+1) \alpha, m+1) .
$$

For simplicity we consider a special case of the vertex operator $Y_{a}(z)$, and let

$$
\begin{aligned}
Y(z) & =Y_{0}(z)=\exp \left(\sum_{n=1}^{\infty} \frac{z^{n}}{n} \alpha h_{-n}\right)=\sum Y_{-n} z^{n}, \\
Y^{*}(z) & =\exp \left(\sum_{n=1}^{\infty} \frac{z^{-n}}{n} \alpha h_{n}\right)=\sum Y_{n}^{*} z^{n} .
\end{aligned}
$$

We remark that one can also use the operator $Y_{a}(z),(a \neq 0)$ in place of $Y(z)$, and most proofs will remain the same.

We note that when $\alpha=1$ the vertex operator $X(z)$ differs from the Schur vertex operator [4] or its truncated form Bernstein operator for Schur functions.

To simplify the notations, for partition $\lambda=\left(\lambda_{1}, \ldots, \lambda_{s}\right)$, we denote the product $X_{-\lambda_{1}} \cdots X_{-\lambda_{s}}$ simply as $X_{-\lambda}$, and similarly for $Y_{-\lambda}$.

We will first give an explicit formula for the vertex operator products. For this purpose, we need the following

Lemma 3.1 For the creation part and annihilation part of $X(z)$ we have

$$
\exp \left(\sum_{n=1}^{\infty} \frac{z^{n}}{n} \alpha h_{-n}\right)=\sum_{\lambda \in \mathcal{P}} z_{\lambda}^{-1} \alpha^{l(\lambda)} h_{-\lambda} z^{|\lambda|}
$$

and

$$
\exp \left(\sum_{n=1}^{\infty} \frac{z^{-n}}{-n} 2 \alpha h_{n}\right) \cdot h_{-\lambda}=\sum_{\mu \subset^{\prime} \lambda}\left(\begin{array}{l}
m(\lambda) \\
m(\mu)
\end{array}\right)(-2)^{l(\mu)} z^{-|\mu|} h_{-\lambda \backslash \mu}
$$

for any partition $\lambda$,

Proof The first one is a direct computation:

$$
\begin{aligned}
\exp \left(\sum_{n=1}^{\infty} \frac{z^{n}}{n} \alpha h_{-n}\right) & =\prod_{n \geq 1} \exp \left(\frac{z^{n}}{n} \alpha h_{-n}\right)=\prod_{n \geq 1} \sum_{i \geq 0} \frac{1}{i !} \frac{z^{n i}}{n^{i}} \alpha^{i} h_{-n}^{i} \\
& =\sum_{\lambda \in \mathcal{P}} z_{\lambda}^{-1} \alpha^{l(\lambda)} h_{-\lambda} z^{|\lambda|} .
\end{aligned}
$$

For the second one, let $h_{n}^{(i)}=h_{n}^{i} / i$. The Heisenberg canonical commutation relation implies that

$$
h_{n}^{(i)} \cdot h_{-n}^{m}=\left(\frac{n}{\alpha}\right)^{i}\left(\begin{array}{c}
m \\
i
\end{array}\right) h_{-n}^{m-i} .
$$


Using this we have

$$
\begin{aligned}
\exp \left(\frac{z^{-n}}{-n} 2 \alpha h_{n}\right) \cdot h_{-n}^{m} & =\sum_{i \geq 0}\left(\frac{2 \alpha z^{-n}}{-n}\right)^{i} h_{n}^{(i)} \cdot h_{-n}^{m} \\
& =\sum_{i \geq 0} z^{-n i}(-2)^{i}\left(\begin{array}{c}
m \\
i
\end{array}\right) h_{-n}^{m-i} .
\end{aligned}
$$

For $\lambda=\left(1^{m_{1}} 2^{m_{2}} \cdots\right)$, we have

$$
\begin{aligned}
& \exp \left(\sum_{n=1}^{\infty} \frac{z^{-n}}{-n} 2 \alpha h_{n}\right) \cdot h_{-\lambda} \\
& =\prod_{n \geq 1} \exp \left(\frac{z^{-n}}{-n} 2 \alpha h_{n}\right) \cdot h_{-1}^{m_{1}} h_{-2}^{m_{2}} \ldots \\
& =\prod_{n \geq 1}\left(\sum_{i_{n} \geq 0} z^{-n i_{n}}(-2)^{i_{n}}\left(\begin{array}{c}
m_{n} \\
i_{n}
\end{array}\right) h_{-n}^{m_{n}-i_{n}}\right)=\sum_{\mu \in \mathcal{P}}\left(\begin{array}{c}
m(\lambda) \\
m(\mu)
\end{array}\right)(-2)^{l(\mu)} z^{-|\mu|} h_{-\lambda \backslash \mu},
\end{aligned}
$$

where the sum runs through all partitions $\mu \subset^{\prime} \lambda$.

In the following we let $\underline{\lambda}=\left(\lambda^{1}, \lambda^{2}, \ldots, \lambda^{s}\right)$ denote a sequence of partitions $\lambda^{1}, \lambda^{2}, \ldots, \lambda^{s}$.

Theorem 3.2 For integer $s \geq 1$, we have

$$
\begin{aligned}
& X_{-\lambda_{s}} \cdots X_{-\lambda_{1}} \cdot e^{n h} \\
& \quad=\sum_{\underline{\mu}, \underline{\underline{v}}} \prod_{i=1}^{s} \frac{(-2 \alpha)^{l\left(v^{i}\right)}}{z_{\nu^{i}}}\left(\begin{array}{c}
m\left(\mu^{i-1}\right) \\
m\left(\mu^{i} \backslash v^{i}\right)
\end{array}\right) \frac{h_{-\mu^{s}}}{(-2)^{l\left(\mu^{s}\right)}} \otimes e^{(n+s) h},
\end{aligned}
$$

where the sum is over $\underline{\mu}=\left(\mu^{1}, \mu^{2}, \ldots, \mu^{s}\right)$ and $\underline{v}=\left(v^{1}, v^{2}, \ldots, v^{s}\right)$ such that $v^{i} \subset^{\prime} \mu^{i},\left|\mu^{i}\right|=\lambda_{1}+\cdots+\lambda_{i}-i(2 n+i) \alpha, \mu^{i} \backslash v^{i} \subset^{\prime} \mu^{i-1}, \mu^{0}=(0)$.

Proof We use induction on $s$. Note that $v^{1}=\mu^{1}$, it is trivial for the case of $s=1$. Applying the annihilation part and the middle term of $X(z)$ to (3.5), we find the right side

$$
\begin{aligned}
& \sum_{\mu \mathcal{C}^{\prime} \mu^{s}} \sum_{\mu, \underline{v}} \prod_{i=1}^{s} \frac{(-2 \alpha)^{l\left(v^{i}\right)}}{z_{\nu^{i}}}\left(\begin{array}{c}
m\left(\mu^{i-1}\right) \\
m\left(\mu^{i} \backslash \nu^{i}\right)
\end{array}\right) \\
& \cdot \frac{(-2)^{l(\mu)}}{(-2)^{l\left(\mu^{s}\right)}}\left(\begin{array}{c}
m\left(\mu^{s}\right) \\
m(\mu)
\end{array}\right) \frac{z^{(2 n+2 s+1) \alpha}}{z^{|\mu|}} h_{-\mu^{s} \backslash \mu} e^{(n+s+1) h} .
\end{aligned}
$$


Replacing $\mu$ with $\mu_{s} \backslash \mu$, it becomes

$$
\begin{aligned}
& \sum_{\mu \subset^{\prime} \mu^{s}} \sum_{\mu, \underline{v}} \prod_{i=1}^{s} \frac{(-2 \alpha)^{l\left(v^{i}\right)}}{z_{v^{i}}}\left(\begin{array}{c}
m\left(\mu^{i-1}\right) \\
m\left(\mu^{i} \backslash v^{i}\right)
\end{array}\right) \\
& \cdot(-2)^{-l(\mu)}\left(\begin{array}{c}
m\left(\mu^{s}\right) \\
m(\mu)
\end{array}\right) \frac{z^{(2 n+2 s+1) \alpha}}{z^{\left|\mu^{s} \backslash \mu\right|} h_{-\mu} e^{(n+s+1) h} .}
\end{aligned}
$$

Thus, we have

$$
\begin{aligned}
X_{-\lambda_{s+1}} X_{-\lambda_{s}} \cdots X_{-\lambda_{1}} . e^{n h}= & \sum_{v} \sum_{\mu \subset^{\prime} \mu^{s}} \sum_{\mu, \underline{v}} \prod_{i=1}^{s} \frac{(-2 \alpha)^{l\left(v^{i}\right)}}{z_{v^{i}}}\left(\begin{array}{c}
m\left(\mu^{i-1}\right) \\
m\left(\mu^{i} \backslash v^{i}\right)
\end{array}\right) \\
& \cdot(-2)^{-l(\mu)}\left(\begin{array}{c}
m\left(\mu^{s}\right) \\
m(\mu)
\end{array}\right) \frac{\alpha^{l(v)}}{z_{v}} h_{-\mu \cup v} e^{(n+s+1) h},
\end{aligned}
$$

where in the first sum $\nu$ is restricted to: $|\nu|-\left|\mu^{s} \backslash \mu\right|=\lambda_{s+1}-(2 n+2 s+1) \alpha$. Changing variables $v=v^{s+1}, \mu=\mu^{s+1} \backslash v^{s+1}$, we finish the proof.

Note that a necessary condition for $X_{-\lambda_{s}} \cdots X_{-\lambda_{1}} . e^{n h} \neq 0$ is that $k_{i}=\lambda_{1}+\cdots+$ $\lambda_{i}-i(2 n+i) \alpha \geq 0$ for $i=1,2, \ldots, s$.

\subsection{Generalized Jacobi-Trudi theorem}

The generalized homogeneous symmetric function with parameter $\alpha$ can be written as follows. For $n \in \mathbb{Z}$,

$$
H_{n}\left(\alpha^{-1}\right)=\sum_{\lambda \vdash n} \alpha^{(\lambda)} z_{\lambda}^{-1} h_{-\lambda}
$$

Its image under $T$ is $Q_{n}(\alpha)$ by (2.2). Clearly one has $Y_{a}(-n) .1=H_{n}\left(\alpha^{-1}\right)$. Notice that by definition $H_{n}\left(\alpha^{-1}\right)=0$ for $n<0$. Combining these,we have the following statement after a simple computation (see also Lemma 2.1).

Lemma 3.3 For any positive integer s, we have

$$
Y\left(z_{1}\right) \cdots Y\left(z_{s}\right)=\sum_{n_{1} \geq 0, \ldots, n_{s} \geq 0} H_{n_{1}}\left(\alpha^{-1}\right) \cdots H_{n_{s}}\left(\alpha^{-1}\right) z_{1}^{n_{1}} \cdots z_{s}^{n_{s}} .
$$

In particular, $Y_{-r_{1}} \cdots Y_{-r_{s}} \cdot 1=H_{r_{1}}\left(\alpha^{-1}\right) \cdots H_{r_{s}}\left(\alpha^{-1}\right)$ for any integers $r_{1}, \ldots, r_{s}$. Moreover, for partition $\lambda$

$$
T\left(Y_{-\lambda} \cdot 1\right)=q_{\lambda}\left(\alpha^{-1}\right)
$$

Thus the vectors $\left\{Y_{-\lambda} e^{m h}\right\}\left(\lambda \in \mathcal{P}\right.$ and $\left.m \in \frac{1}{2} \mathbb{Z}\right)$ form a linear basis in the representation space $V$, corresponding to the basis of generalized homogeneous polynomials. 
To proceed further we define the normalization of vertex operators, which helps to separate the singular part. The normalization of $X\left(z_{1}\right) \cdots X\left(z_{s}\right)$ is defined as

$$
\begin{aligned}
& : X\left(z_{1}\right) \cdots X\left(z_{s}\right): \\
& \quad=\exp \left(\sum_{n=1}^{\infty} \frac{z_{1}^{n}+\cdots+z_{s}^{n}}{n} \alpha h_{-n}\right) \exp \left(\sum_{n=1}^{\infty} \frac{z_{1}^{-n}+\cdots+z_{s}^{-n}}{-n} 2 \alpha h_{n}\right) A,
\end{aligned}
$$

where $A=e^{s h} \exp \left(2 \alpha\left(\ln z_{1}+\cdots+\ln z_{s}\right) \partial_{h}\right)\left(z_{1} \cdots z_{s}\right)^{\alpha}$. Similarly when the normal product is taken on mixed product of $X(z), Y(z)$, and $Y^{*}(z)$, one always moves the annihilation operators to the right.

We define the lowering operator $D_{i}$ on the bases of symmetric functions by

$$
D_{i}\left(H_{\lambda}\right)=H_{\lambda_{1}} \cdots H_{\lambda_{i-1}} H_{\lambda_{i}-1} H_{\lambda_{i+1}} \cdots H_{\lambda_{l}} \text {. }
$$

The rising operator is defined by $R_{i j}=D_{i}^{-1} D_{j}$. Like the raising operator $R_{i j}$ the lowering operator $D_{i}$ is not always invertible, one needs to make sure that each application of $D_{i}$ is non-zero.

Lemma 3.4 For $s \geq 1$, we have

$$
\begin{aligned}
X_{-\lambda_{1}} X_{-\lambda_{2}} \cdots X_{-\lambda_{s}} \cdot e^{n h}= & \left(\prod_{1 \leq i<j \leq s}\left(D_{i}-D_{j}\right)^{2 \alpha} \cdot \prod_{1 \leq i \leq s} D_{i}^{\left(n+\frac{1}{2}\right) 2 \alpha}\right) \\
& \cdot H_{\lambda_{1}}\left(\alpha^{-1}\right) \cdots H_{\lambda_{s}}\left(\alpha^{-1}\right) \otimes e^{(n+s) h} .
\end{aligned}
$$

Proof Observe that for $|w|<|z|$

$$
\begin{aligned}
& \exp \left(\sum_{n=1}^{\infty} \frac{z^{-n}}{-n} 2 \alpha h_{n}\right) \exp \left(\sum_{n=1}^{\infty} \frac{w^{n}}{n} \alpha h_{-n}\right) \\
& \quad=\exp \left(\sum_{n=1}^{\infty} \frac{w^{n}}{n} \alpha h_{-n}\right) \exp \left(\sum_{n=1}^{\infty} \frac{z^{-n}}{-n} 2 \alpha h_{n}\right)\left(1-\frac{w}{z}\right)^{2 \alpha} .
\end{aligned}
$$

Using induction on $s$ and the normalization we have

$$
X\left(z_{1}\right) \cdots X\left(z_{s}\right)=\prod_{1 \leq i<j \leq s}\left(z_{i}-z_{j}\right)^{2 \alpha}: X\left(z_{1}\right) \cdots X\left(z_{s}\right):
$$

Applying the action on $e^{n h}$, we have

$$
\begin{aligned}
X\left(z_{1}\right) \cdots X\left(z_{s}\right) \cdot e^{n h}= & \prod_{1 \leq i<j \leq s}\left(z_{i}-z_{j}\right)^{2 \alpha} \prod_{i=1}^{s} z_{i}^{\left(n+\frac{1}{2}\right) 2 \alpha} \\
& \cdot \prod_{i=1}^{s}\left(\sum_{n \geq 0} H_{n}\left(\alpha^{-1}\right) z_{i}^{n}\right) \otimes e^{(n+s) h},
\end{aligned}
$$


where $\left(z_{i}-z_{j}\right)^{2 \alpha}=z_{i}^{2 \alpha}\left(1-z_{j} / z_{i}\right)^{2 \alpha}$ if there is an expansion. Taking the coefficient of $z^{\lambda}$ we obtain the statement.

In concern with the operator in Lemma 3.4, we have the following result on the square of Vandermonde determinant.

Lemma 3.5 Let $V\left(X_{s}\right)=V\left(x_{1}, \ldots, x_{s}\right)=\prod_{1 \leq i<j \leq s}\left(x_{i}-x_{j}\right), s \geq 2$. For $V\left(X_{s}\right)^{2}$, the coefficient of the term $\prod_{i=1}^{s} x_{i}^{s-1}$ is $(-1)^{s(s-1) / 2} s$ !, and the coefficient of $x_{k} x_{s}^{-1} \prod_{i=1}^{s} x_{i}^{s-1}(k=1, \ldots, s-1)$ is $-(-1)^{s(s-1) / 2}(s-1) !$.

Proof The Vandermonde determinant $V\left(X_{s}\right)$ is the determinant of $M=\left(x_{j}^{i-1}\right)_{s \times s}$. Then $V\left(X_{s}\right)^{2}=\operatorname{det}\left(M M^{T}\right)=\operatorname{det}\left(p_{i+j-2}\right)_{s \times s}$, where $p_{n}=x_{1}^{n}+\cdots+x_{s}^{n}$. The product $\prod_{1 \leq i \leq s} x_{i}^{s-1}$ only appears in the minor-diagonal term $p_{s-1} p_{s-1} \cdots p_{s-1}=$ $p_{s-1}^{s}$ of the determinant, thus the coefficient is $(-1)^{s(s-1) / 2} s !$. Similarly the term $x_{k} x_{s}^{-1} \prod_{1 \leq i \leq s} x_{i}^{s-1}(k=1, \ldots, s-1)$ only appears in the term of the form $p_{s-2} p_{s} p_{s-1}^{s-2}$ of the determinant, so the coefficient is $-(-1)^{s(s-1) / 2}(s-1)(s-2)$ !.

For any partition $\lambda$ and a fixed parameter $\alpha$, we set

$$
H_{\lambda}(\alpha)=H_{\lambda_{1}}(\alpha) \cdots H_{\lambda_{l}}(\alpha)
$$

Clearly the set of vectors $H_{\lambda}\left(\alpha^{-1}\right) e^{m h}$ forms an $\mathbb{C}$-basis of the vertex operator space $V$. Under the map $T$, the vector $H_{\lambda}\left(\alpha^{-1}\right)$ is the symmetric function $q_{\lambda}\left(\alpha^{-1}\right)$. For fixed $\alpha \in \mathbb{N}$ and $m \in \frac{1}{2} \mathbb{Z}$, we define $\mathcal{P}_{\alpha, m}$ to be the set of partitions $\lambda$ such that $\lambda_{i}-\lambda_{i+1} \geq 2 \alpha$ and $\lambda_{l(\lambda)} \geq(2 m+1) \alpha$. The following result is a generalization of Jacobi-Trudi theorem for our vertex operator basis.

Theorem 3.6 Let $\alpha \in \mathbb{N}, m \in \frac{1}{2} \mathbb{Z}$. The set of products $X_{-\lambda} e^{m h}\left(\lambda \in \mathcal{P}_{\alpha, m}, m \in \mathbb{Z}\right)$ forms a $\mathbb{C}$-basis in the vertex algebra $V$. Moreover one has, for a partition $\lambda$ of length $l$ and $\lambda_{l} \geq(2 m+1) \alpha$,

$$
X_{-\lambda} e^{m h}=\sum_{\mu \geq \lambda} a_{\lambda \mu}\left(\alpha^{-1}\right) H_{\mu-(2 m+1) \alpha \mathbf{1}-2 \alpha \delta} e^{(m+l(\lambda)) h},
$$

where $\mu$ runs through the l-tuple compositions such that $|\mu|=|\lambda|$, and $\mathbf{1}=$ $(1, \ldots, 1) \in \mathbb{N}^{l}$ and $\delta=(l-1, l-2, \ldots, 1,0)$. If $\lambda \in \mathcal{P}_{\alpha, m}$, the leading coefficient $a_{\lambda \lambda}\left(\alpha^{-1}\right)=1$, and the degree of $X_{-\lambda} e^{m h}$ is $(|\lambda|-\alpha(2 m+l) l, m+l)$.

Proof For any partition $\lambda$ of length $l$, we can rewrite Lemma 3.4 in terms of raising operators.

$$
\begin{gathered}
X_{-\lambda_{1}} X_{-\lambda_{2}} \cdots X_{-\lambda_{l}} \cdot e^{m h}=\left(\prod_{1 \leq i<j \leq l}\left(1-R_{i j}\right)^{2 \alpha} \cdot \prod_{1 \leq i \leq l} D_{i}^{\left(m+l-i+\frac{1}{2}\right) 2 \alpha}\right) \\
\cdot H_{\lambda_{1}}\left(\alpha^{-1}\right) \cdots H_{\lambda_{l}}\left(\alpha^{-1}\right) \otimes e^{(m+l) h}
\end{gathered}
$$


The raising operators map $H_{\lambda}$ into $H_{\mu}$ with $\mu \geq \lambda$, and the product

$$
\prod_{1 \leq i<j \leq l}\left(1-R_{i j}\right)^{2 \alpha}=1+\sum_{e \neq 0} \pm \prod_{i<j} R_{i j}^{e_{i j}},
$$

where $e_{i j}$ are non-negative exponents, so one obtains the expansion of $X_{-\lambda} e^{m h}$ into the right-hand side of (3.8). Note that when $\lambda_{l} \geq(2 m+1) \alpha$ and $\lambda_{i}-\lambda_{i+1} \geq 2 \alpha$ ( $i=0, \ldots, l-1)$, the leading term $H_{\lambda-(2 m+1) \alpha 1-2 \alpha \delta} e^{(m+l(\lambda)) h}$ is non-zero. As $e \neq 0$, all the remaining terms in the expansion differ from the leading term, which shows that the representation matrix of the set $X_{-\lambda} e^{m h}\left(\lambda \in \mathcal{P}_{\alpha, m}\right)$ in terms of the basis $H_{\mu} e^{(m+l(\lambda)) h}$ (of fixed degree and length) is triangular and has ones on the diagonal. This implies that the basis element $H_{\mu} e^{n h}$ (of fixed degree and length $l$ ) can be expressed as a linear combination of $X_{-\lambda} e^{(n-l) h}$, where $\lambda \geq \mu+(2 n-2 l+1) \alpha \mathbf{1}+2 \alpha \delta$, i.e. $\lambda \in \mathcal{P}_{\alpha, n-l}$. Hence the set $X_{-\lambda} e^{m h}\left(\lambda \in \mathcal{P}_{\alpha, m}\right)$ forms a basis of the vertex operator algebra.

We will see that in certain cases the vectors of type $X_{-\lambda} e^{m h}$ are actually Jack symmetric functions.

\subsection{Jack functions of rectangular shapes}

We first look at combinatorial properties of rectangular and marked rectangular partitions.

Lemma 3.7 Let $\lambda=\left((k+1)^{s},(k)^{t}\right)$ be a partition with $t \in \mathbb{Z}_{>0}, k, s \in \mathbb{Z}_{\geq 0}$. Then for any partition $\mu$ satisfying $|\mu|=|\lambda|$ and $l(\mu) \leq l(\lambda)$, we have $\mu \geq \lambda$.

Proof Let $\lambda=\left(\lambda_{1}, \ldots, \lambda_{s+t}\right)$. If $s=0$ it is obviously true. So we can assume that $s \geq 1$. Let $\mu=\left(\mu_{1}, \ldots, \mu_{s+t}\right)$ be another partition with $\mu_{1} \geq \mu_{2} \geq \cdots \geq \mu_{s+t} \geq 0$. The assumption says that $\mu_{1} \geq k+1$. If we do not have $\mu \geq \lambda$, there should be an $r$, $1 \leq r<s+t$ such that

$$
\sum_{i=1}^{r} \mu_{i} \geq \sum_{i=1}^{r} \lambda_{i}
$$

and

$$
\sum_{i=1}^{r+1} \mu_{i}<\sum_{i=1}^{r+1} \lambda_{i}
$$

It then follows that $k \geq \mu_{r+2}, \mu_{r+3}, \ldots$, but $k \leq \lambda_{r+2}, \lambda_{r+3}, \ldots$ Subsequently

$$
\sum_{i=1}^{s+t} \mu_{i}<\sum_{i=1}^{s+t} \lambda_{i}
$$

a contradiction with $|\mu|=|\lambda|$. 
Next we consider the mixed products of vertex operators. The following result is an easy computation by vertex operator calculus.

Lemma 3.8 The operator product expansion of mixed products is given by

$$
\begin{aligned}
& Y^{*}\left(w_{1}\right) \cdots Y^{*}\left(w_{t}\right) X\left(z_{1}\right) \cdots X\left(z_{s}\right) \\
& \quad=:-: \prod_{1 \leq i<j \leq s}\left(z_{i}-z_{j}\right)^{2 \alpha} \prod_{j=1}^{s} \prod_{i=1}^{t}\left(1-z_{j} w_{i}\right)^{-\alpha},
\end{aligned}
$$

where : - : denotes : $Y^{*}\left(w_{1}\right) \cdots Y^{*}\left(w_{t}\right) X\left(z_{1}\right) \cdots X\left(z_{s}\right):$.

For any partition $\lambda$, we observe that the degree of the vector $X_{-\lambda} e^{-\frac{l(\lambda)}{2} h}$ is $(|\lambda|, l(\lambda) / 2)$ (see Theorem 3.6). Now we can prove the main theorem.

Theorem 3.9 For partition $\lambda=\left((k+1)^{s},(k)^{t}\right)$ with $k \in \mathbb{Z}_{\geq 0}, s \in \mathbb{Z}_{>0}, t \in\{0,1\}$, we have

$$
T\left(X_{-\lambda} e^{-(s+t) h / 2}\right)=c(\alpha) Q_{\lambda}\left(\alpha^{-1}\right),
$$

where $c(\alpha)$ is a rational function of $\alpha$, and $c(1)=(-1)^{s(s-1+2 t) / 2} s !$.

We remark that when $\lambda$ is a rectangular Young diagram (i.e. $t=0$ ) the result was first proved by Mimachi and Yamada [11] using differential operators. When $s=t=1$, it was proved in [7]. Another interesting phenomenon is that when $\alpha=1$, we obtain a new vertex operator formula for the rectangular shapes and marked rectangular shapes. In [12] Schur functions of rectangular shapes are proved to be singular vectors for the Virasoro algebra $(\alpha=1)$.

Proof For $\lambda=\left((k+1)^{s} k^{t}\right)$, let $u$ denote $T\left(X_{-\lambda} \cdot e^{-(s+t) h / 2}\right)$. Note that $u$ is a linear combination of $Q_{\mu}\left(\alpha^{-1}\right)$ 's with $\mu \geq \lambda$ by Lemmas 3.4 and 2.1. By Lemma 3.7, we need to show that $u$ is orthogonal to $Q_{\mu}\left(\alpha^{-1}\right)$, for all $\mu$ such that $\mu \vdash k t+s(k+1)$ and $\mu \neq \lambda$. This will be done in the following. As for the coefficient, we have applied Lemmas 3.5 and 2.1 as well as Lemma 3.4, which confirms that $u$ is non-zero and the coefficient $c(\alpha)$ satisfies the given formula.

To prove the orthogonality, consider $\mu$ in two cases:

(1) By Corollary 3.7 and Lemma 2.1, for $\mu<\lambda$, or $\mu$ is incomparable with $\lambda$, it follows easily that $u$ is orthogonal to $Q_{\mu}\left(\alpha^{-1}\right)$.

(2) If partition $\mu=\left(m_{1}, \ldots, m_{r}\right)$ and $\mu>\lambda$ (or $\mu$ are incomparable with $\lambda$ ), then it follows that $m_{1}>k+1$. By Lemmas 2.1 and 3.3, to prove that $u$ is orthogonal to $Q_{\mu}\left(\alpha^{-1}\right)$, we only need to see that the following product is zero:

$$
\begin{gathered}
\left\langle\left(X_{-(k+1)}\right)^{s}\left(X_{-k}\right)^{t} \cdot e^{-(s+t) h / 2}, Y_{-m_{r}} \cdots Y_{-m_{1}} \cdot e^{(s+t) h / 2}\right\rangle \\
=\left\langle Y_{-m_{1}}^{*} \cdots Y_{-m_{t}}^{*}\left(X_{-(k+1)}\right)^{s}\left(X_{-k}\right)^{t}, e^{(s+t) h / 2}\right\rangle,
\end{gathered}
$$


which equals to the coefficient of $w_{1}^{-m_{1}} \cdots w_{t}^{-m_{t}}\left(z_{1} \cdots z_{s}\right)^{k+1}\left(z_{s+1} \cdots z_{s+t}\right)^{k}$ in the following expression

$$
\begin{gathered}
\left\langle Y^{*}\left(w_{1}\right) \cdots Y^{*}\left(w_{r}\right) X\left(z_{1}\right) \cdots X\left(z_{s+t}\right) \cdot e^{-(s+t) h / 2}, e^{(s+t) h / 2}\right\rangle \\
=z_{s+t}^{\left(-\frac{s+t}{2}+\frac{1}{2}\right) 2 \alpha} z_{s+t-1}^{\left(-\frac{s+t}{2}+1+\frac{1}{2}\right) 2 \alpha} \cdots z_{1}^{\left(-\frac{s+t}{2}+s+t-1+\frac{1}{2}\right) 2 \alpha} \\
\quad \cdot \prod_{s+t \geq j>i \geq 1}\left(1-z_{j} z_{i}^{-1}\right)^{2 \alpha} \prod_{i=1}^{s+t}\left(1-z_{j} w_{i}^{-1}\right)^{-\alpha} \\
= \pm \prod_{1 \leq i \neq j \leq s+t}\left(1-z_{j} z_{i}^{-1}\right)^{\alpha} \prod_{j=1}^{s+t} \prod_{i=1}^{r}\left(1-z_{j} w_{i}^{-1}\right)^{-\alpha},
\end{gathered}
$$

where we have used Lemma 3.8. In Lemma 3.11, to be proved in the next section, we will see that this coefficient is indeed zero. Hence the theorem is proved.

In general we have the following result.

Corollary 3.10 For partition $\lambda=\left((k+1)^{s},(k)^{t}\right)$ with $k \in \mathbb{Z}_{\geq 0}, s \in \mathbb{Z}_{>0}, t \in\{0,1\}$, and $r \in \mathbb{Z}, \alpha \in \mathbb{Z}_{>0}$ such that $r \alpha \leq k+\delta_{t, 0}$ we have

$$
T\left(X_{-\lambda} e^{-\frac{s+t-r}{2} h}\right)=c Q_{\lambda-r \alpha 1}\left(\alpha^{-1}\right),
$$

where $c$ is a non-zero constant, and $\mathbf{1}=(1,1, \ldots, 1) \in \mathbb{Z}^{s+t}$.

Proof The proof is essentially the same as that of Theorem 3.9. The condition $r \alpha \leq k+\delta_{t, 0}$ is necessary to guarantee $T\left(X_{-\lambda} e^{-\frac{s+t-r}{2} h}\right) \neq 0$ (see Remark of Theorem 3.2).

Lemma 3.11 The expansion of the contraction function

$$
H_{\alpha}\left(Z_{s}, W_{t}\right)=\prod_{s \geq i \neq j \geq 1}\left(1-z_{j} z_{i}^{-1}\right)^{\alpha} \prod_{j=1}^{s} \prod_{i=1}^{t}\left(1-z_{j} w_{i}^{-1}\right)^{-\alpha}
$$

in the region of $\left|z_{j}\right|<\left|w_{i}\right|$ does not contain terms of the form $w_{1}^{-m_{1}} \cdots w_{t}^{-m_{t}} z_{1}^{k_{1}} \cdots z_{s}^{k_{s}}$ where $m_{1}>k_{i}(i=1,2, \ldots, s)$.

\section{Analysis of $H_{\alpha}\left(Z_{s}, W_{t}\right)$}

We have the following lemma to split $H_{\alpha}\left(Z_{s}, W_{t}\right)$ :

Lemma 4.1 For positive integers $r, s, i \neq j$, there are non-negative integers $f_{m}$ and $g_{n}$ such that:

$$
\left(\frac{1-z_{i} z_{j}^{-1}}{1-z_{i} w^{-1}}\right)^{r}\left(\frac{1-z_{j} z_{i}^{-1}}{1-z_{j} w^{-1}}\right)^{s}=\sum_{m=1}^{r}\left(\frac{1-z_{i} z_{j}^{-1}}{1-z_{i} w^{-1}}\right)^{m} f_{m}+\sum_{n=1}^{s}\left(\frac{1-z_{j} z_{i}^{-1}}{1-z_{j} w^{-1}}\right)^{n} g_{n} .
$$


Proof For simplicity we denote $a=\frac{1-z_{i} z_{j}^{-1}}{1-z_{i} w^{-1}}, b=\frac{1-z_{j} z_{i}^{-1}}{1-z_{j} w^{-1}}$, it can be verified directly that $a b=a+b$. Repeatedly using this, we can write $a^{r} b^{s}$ into the wanted form.

Assume first that $\alpha$ is a positive integer. Consider

$$
H_{n}\left(Z_{s}, w\right)=H_{n}\left(Z_{s}, W_{1}\right)=\prod_{s \geq i \neq j \geq 1}\left(1-z_{j} z_{i}^{-1}\right)^{n} \prod_{j=1}^{s}\left(1-z_{j} w^{-1}\right)^{-n},
$$

where we identified $w_{1}$ with $w$ for simplicity. Notice that

$$
H_{n}\left(Z_{s}, W_{t}\right)=H_{n}\left(Z_{s}, w\right) \prod_{i=2}^{t} \prod_{j=1}^{s}\left(1-z_{j} w_{i}^{-1}\right)^{-n} .
$$

If the expansion of $H_{n}\left(Z_{s}, w\right)$ in the region $\left|z_{j}\right|<\left|w_{i}\right|$ does not contain terms of the form $w_{1}^{-1} \cdots w_{t}^{-1} z_{1}^{k_{1}} \cdots z_{s}^{k_{s}}$ with $m_{1}>k_{i}(i=1, \ldots, s)$, neither does $H_{n}\left(Z_{s}, w_{t}\right)$. Thus the following result will imply Lemma 3.11 .

Theorem 4.2 For $n, s \in \mathbb{N}, s \geq 2$, there are polynomials $f_{i, j}$ in $z_{k} z_{l}^{-1}$ 's $(1 \leq k \neq$ $l \leq s)$ such that:

$$
H_{n}\left(Z_{s}, w\right)=\sum_{i=1}^{s} \sum_{j=1}^{n}\left(1-z_{i} w^{-1}\right)^{-j} f_{i, j}
$$

Moreover for each $i$ and $j, f_{i, j}$ is a polynomial in $z_{i}$.

Proof To prove the existence of $f_{i, j}$ 's, we will use induction on $s$.

In the case of $s=2$ it is true by Lemma 4.1. Assume that it holds true for $s$, we have

$$
\begin{aligned}
H_{n}\left(Z_{s+1}, w\right)= & H_{n}\left(Z_{s}, w\right) A_{s+1}=\sum_{i=1}^{s} \sum_{j=1}^{n}\left(1-z_{i} w^{-1}\right)^{-j} A_{s+1} f_{i, j} \\
= & \sum_{i=1}^{s} \sum_{j=1}^{n}\left(1-z_{i} z_{s+1}^{-1}\right)^{j}\left(1-z_{i} w^{-1}\right)^{-j}\left(1-z_{s+1} z_{i}^{-1}\right)^{n} \\
& \cdot\left(1-z_{s+1} w^{-1}\right)^{-n} B_{s+1, i, j} f_{i, j},
\end{aligned}
$$

where

$$
\begin{aligned}
A_{s+1} & =\left(1-z_{s+1} w^{-1}\right)^{-n} \prod_{l=1}^{s}\left(1-z_{l} z_{s+1}^{-1}\right)^{n}\left(1-z_{s+1} z_{l}^{-1}\right)^{n} \\
& =\left(1-z_{i} z_{s+1}^{-1}\right)^{j}\left(1-z_{s+1} z_{i}^{-1}\right)^{n}\left(1-z_{s+1} w^{-1}\right)^{-n} B_{s+1, i, j} .
\end{aligned}
$$

Notice that the term inside the sum can be split by Lemma 4.1, while $B_{s+1, i, j}$ is a product of $\left(1-z_{k} z_{l}^{-1}\right)$ 's, and the existence follows. 
As for the second part, note that $H_{n}(Z, w)$ is symmetric about $z_{1}, \ldots, z_{s}$, we only need to prove that $f_{1, j}(j=1, \ldots, n)$ are polynomials of $z_{1}$. Multiplying two sides of (4.1) by $\left(1-z_{1} w^{-1}\right)^{n} \cdots\left(1-z_{s} w^{-1}\right)^{n}$, we have

$$
\begin{aligned}
& \prod_{1 \leq i \neq j \leq s}\left(1-z_{j} z_{i}^{-1}\right)^{n} \\
& =\sum_{i=1}^{s} \sum_{j=1}^{n}\left(1-z_{1} w^{-1}\right)^{n} \cdots\left(1-z_{i} w^{-1}\right)^{n-j} \cdots\left(1-z_{s} w^{-1}\right)^{n} f_{i, j} .
\end{aligned}
$$

Using induction on $j^{\prime}=n-j$ : first, let $w=z_{1}$ in (4.2), we have

$$
\prod_{1 \leq i \neq j \leq s}\left(1-z_{i} z_{j}^{-1}\right)^{n}=f_{1, n} \prod_{i=2}^{s}\left(1-z_{i} z_{1}^{-1}\right)^{n}
$$

Eliminating the common factor we find,

$$
f_{1, n}=\prod_{i=2}^{s}\left(1-z_{1} z_{i}^{-1}\right)^{n} \prod_{2 \leq i \neq j \leq s}\left(1-z_{i} z_{j}^{-1}\right)^{n}
$$

which implies the case $j^{\prime}=0$. Assume that it's true for $j^{\prime}<r$. Let $j^{\prime}=r \leq n-1$. Differentiating both sides of (4.2) with respect to $z_{1}$, and set $w=z_{1}$, we have:

$$
\frac{\partial^{r}}{\partial z_{1}^{r}} \prod_{1 \leq i \neq j \leq s}\left(1-z_{i} z_{j}^{-1}\right)^{n}=\prod_{i=2}^{s}\left(1-z_{i} / z_{1}\right)^{n} \cdot \sum_{i=0}^{r}\left(\begin{array}{l}
r \\
i
\end{array}\right) i !\left(-z_{1}^{-1}\right)^{i} \frac{\partial^{r-i}}{\partial z_{1}^{r-i}} f_{1, n-i} .
$$

The term $i=r$ in the sum contains $f_{1, n-r}$ and one finds that,

$$
\begin{aligned}
f_{1, n-r}= & (r !)^{-1}\left(-z_{1}\right)^{r} \prod_{i=2}^{s}\left(1-z_{i} / z_{1}\right)^{-n} \frac{\partial^{r}}{\partial z_{1}^{r}} \prod_{1 \leq i \neq j \leq s}\left(1-z_{i} z_{j}^{-1}\right)^{n} \\
& -\sum_{i=0}^{r-1}((r-i) !)^{-1}\left(-z_{1}\right)^{r-i} \frac{\partial^{r-i}}{\partial z_{1}^{r-i}} f_{1, n-i} .
\end{aligned}
$$

Note that

$$
\begin{aligned}
& \frac{\partial^{r}}{\partial z_{1}^{r}} \prod_{1 \leq i \neq j \leq s}\left(1-z_{i} z_{j}^{-1}\right)^{n} \\
& \quad=\prod_{2 \leq i \neq j \leq s}\left(1-z_{i} z_{j}^{-1}\right)^{n} \sum c\left(a_{i}, b_{i}\right) \prod_{i=2}^{s} \frac{\partial^{a_{i}}}{\partial z_{1}^{a_{i}}}\left(\left(1-z_{1} / z_{i}\right)^{n}\right) \frac{\partial^{b_{i}}}{\partial z_{1}^{b_{i}}}\left(\left(1-z_{i} / z_{1}\right)^{n}\right),
\end{aligned}
$$

where the sum is over vectors $\left(a_{2} \cdots a_{s}, b_{2}, \ldots, b_{s}\right)$ with nonnegative integer components which sum up to $r$, and $c\left(a_{i}, b_{i}\right)=r ! /\left(a_{2} ! \cdots a_{s} ! b_{2} ! \cdots b_{s} !\right)$. Now the first part 
of $f_{1, n-r}$ is

$$
\begin{aligned}
& (r !)^{-1}(-1)^{r} z_{1}^{r-b_{2}-\cdots-b_{s}} \prod_{2 \leq i \neq j \leq s}\left(1-z_{i} z_{j}^{-1}\right)^{n} \\
& \cdot \sum c\left(a_{i}, b_{i}\right) \prod_{i=2}^{s} \frac{\partial^{a_{i}}}{\partial z_{1}^{a_{i}}}\left(\left(1-z_{1} / z_{i}\right)^{n}\right) \frac{\partial^{b_{i}}}{\partial z_{1}^{b_{i}}}\left(\left(1-z_{i} / z_{1}\right)^{n}\right)\left(1-z_{i} z_{1}^{-1}\right)^{-n} z_{1}^{b_{i}} .
\end{aligned}
$$

By the following lemma and the assumption of induction, $\lim _{z_{1} \rightarrow 0} f_{1, n-r}$ exists if $z_{i} \neq 0$. Observe that $f_{1, n-r}$ is polynomial of $z_{k} z_{l}^{-1}$, s, it should be a polynomial of $z_{1}$ as well.

Lemma 4.3 For $a \neq 0$, let $g_{s, m}(z)=z^{s}(1-a / z)^{-m} \frac{\partial^{s}}{\partial z^{s}}(1-a / z)^{m}$, then $\lim _{z \rightarrow 0} g_{s, m}(z)$ exists for $0 \leq s<m$.

Proof We use induction on $s$ again. The initial step is trivial. Consider $s+1<m$,

$$
\begin{aligned}
g_{s+1, m}(z) & =z^{s+1}(1-a / z)^{-m} \frac{\partial^{s}}{\partial z^{s}}\left(m(1-a / z)^{m-1} a z^{-2}\right) \\
& =z^{s+1}(1-a / z)^{-m} a m \sum_{i=0}^{s} d_{i} z^{-(2+s-i)} \frac{\partial^{i}}{\partial z^{i}}(1-a / z)^{m-1} \\
& =\sum_{i=0}^{s} c_{i} g_{i, m-1}(z)(z-a)^{-1},
\end{aligned}
$$

where $c_{i}=\operatorname{am}\left(\begin{array}{c}s \\ i\end{array}\right)(1+s-i) !(-1)^{s-i}=a m d_{i}$, and the lemma follows.

\section{References}

1. Feigin, B., Feigin, E.: Principal subspace for the bosonic vertex operator $\phi_{\sqrt{2 m}}(z)$ and Jack polynomials. Adv. Math. 206(2), 307-328 (2006)

2. Garsia, A.: Orthogonality of Milne's polynomials and raising operators. Discrete Math. 99(1-3), 247$264(1992)$

3. Jack, H.: A class of symmetric polynomials with a parameter. Proc. R. Soc. Edinb. Sect. A 69, 1-18 $(1970 / 1971)$

4. Jing, N.: Vertex operators, Vertex operators, symmetric functions, and the spin group $\Gamma_{n}$. J. Algebra 138(2), 340-398 (1991)

5. Jing, N.: Vertex operators and Hall-Littlewood symmetric functions. Adv. Math. 87(2), 226-248 (1991)

6. Jing, N.: $q$-hypergeometric series and Macdonald functions. J. Algebr. Comb. 3, 291-305 (1994)

7. Jing, N., Józefiak, T.: A formula for two row Macdonald functions. Duke Math. J. 67(2), 377-385 (1992)

8. Lepowsky, J., Primc, M.: Structure of the Standard Modules for the Affine Lie Algebra $A_{1}^{(1)}$. Cont. Math., vol. 46. Am. Math. Soc., Providence (1985)

9. Lepowsky, J., Wilson, R.: The structure of standard modules. I. Universal algebras and the RogersRamanujan identities. Invent. Math. 77(2), 199-290 (1984)

10. Macdonald, I.G.: Symmetric Unctions and Hall Polynomials, 2nd edn. With Contributions by A. Zelevinsky. Oxford Univ. Press, New York (1995) 
11. Mimachi, K., Yamada, Y.: Singular vectors of the Virasoro algebra in terms of Jack symmetric polynomials. Commun. Math. Phys. 174(2), 447-455 (1995)

12. Wakimoto, M., Yamada, H.: Irreducible decompositions of representations of the Virasoro algebra. Lett. Math. Phys. 7(6), 513-516 (1983)

13. Stanley, R.: Some combinatorial properties of Jack symmetric functions. Adv. Math. 77(1), 76-115 (1989)

14. Zabrocki, M.: A Macdonald vertex operator and standard tableaux statistics for the two-column $(q, t)$ Kostka coefficients. Electron. J. Comb. 5, 45 (1998)

15. Zelevinsky, A.: Representations of Finite Classical Groups. Lecture Notes in Math., vol. 869. Springer, Berlin-New York (1981) 